\title{
Método para Avaliação do Grau de Maturidade Tecnológica no Processo de Desenvolvimento de Produtos da Indústria Metal-Mecânica
}

\author{
Almiro M. Silva Neto \& Luís G. Trabasso
}

O Serviço Nacional de Aprendizagem Industrial (SENAI) presta serviços técnicos para suprir demandas em desenvolvimento de produtos por meio de seus Institutos SENAI de Tecnologia (ISTs). O objetivo deste trabalho é propor um método para avaliação do grau de maturidade no Processo de Desenvolvimento de Produtos (PDP), fundamentado na escala Technology Readiness Level (TRL), para ser aplicado em empresas do complexo metal-mecânico. Foi desenvolvida a escala New Product Development Readiness Level (NPDRL) para avaliar o grau de maturidade do PDP com vistas a encaminhar orientações para planos de ação para execução de serviços pelos ISTs goianos.

Palavras-chave: Processo de Desenvolvimento de Produtos; Nível de maturidade em desenvolvimento de produtos; TRL.

The SENAI (Serviço Nacional de Aprendizagem Industrial) delivers technical services to fulfill demands in product development through its SENAI Institutes of Technology (ISTs). The goal of this paper is to propose a method for measuring the degree of maturity in the product development process, based on the Technology Readiness Level (TRL) scale and apply it in an industrial metal-mechanic complex. It has been developed a New Product Development Readiness Level (NPDRL) scale to evaluate the degree of maturity of the PDP in order to submit guidelines for action plans to be proposed during the execution of services by Goiás State ISTs, Brazil.

Palavras-chave: New Product Development; Maturity level in product development; TRL. 


\section{Introdução}

Reconhece-se a importância da estruturação do Processo de Desenvolvimento de Produtos (PDP) para o favorecimento e ampliação da competitividade de uma empresa. Além de ocupar-se com atividades que vão desde a percepção de uma oportunidade de mercado até a entrega do produto (e eventual retirada do mercado), ele auxilia no desenvolvimento de produtos com maior valor agregado, auxilia na redução de prazos para lançamentos de produto e ainda pode reduzir custos finais de produção ${ }^{1 ; 2 ; 3}$. Dada sua extensão em aplicações, o PDP faz-se presente nos diversos setores industriais e entre estes destaca-se o complexo industrial metalmecânico. Kamisnki et al. ${ }^{4}$ considera que este complexo industrial é um dos mais importantes da indústria brasileira pois gera emprego e renda em todo o país. No estado de Goiás, receberá aproximadamente $10 \%(\mathrm{R} \$ 3,45$ bilhões) do total da intenção de investimentos em indústrias até o ano $2017^{5}$.

Ao buscar informações sobre como as empresas goianas do complexo metal-mecânico (dada a importância desse complexo para todo o setor industrial) desenvolvem seus produtos (dada que essa é uma temática de preocupação em relação ao aumento de competitividade industrial e, por consequência, dos ISTs - Institutos SENAI de Tecnologia), isto é, como e quais métodos, técnicas e ferramentas utilizam, deparou-se com insuficiência de informações para direcionar as atividades voltadas à prestação de serviços em PDP por parte dos ISTs. Assim, de acordo com o cenário explanado, este trabalho é motivado para a estruturação de um método que permita gerar entendimento de como as empresas goianas do complexo industrial metal-mecânico desenvolvem seus produtos.

Assim, o objetivo deste trabalho é propor um método para avaliar o grau de maturidade no PDP, fundamentado na escala TRL (Technology Readiness Level), de forma a possibilitar a geração de conhecimento sobre o grau de maturidade do PDP em indústrias do complexo metalmecânico e assim indicar um norteador para o encaminhamento de orientações para planos de ação a serem propostos durante a execução de possíveis serviços dos ISTs goianos.

\section{Fundamentação Teórica}

\section{PROCESSO DE DESENVOLVIMENTO DE PRODUTOS}

As atividades produtivas acompanham a evolução das atividades humanas, no entanto, o conceito do processo de projeto de produto apenas tornou-se uma disciplina independente a partir da década de 1960 . Nesse período, perceberam-se o crescimento do volume de produção e a necessidade de superar a concorrência com o estabelecimento da globalização dos mercados ${ }^{1}$. Nesse sentido, Ulrich e Eppinger ${ }^{3}$ consideram que o sucesso econômico de uma organização dá-se pela habilidade na identificação de valores e necessidades dos consumidores e produção rápida e a baixos custos de produtos para suprir essas demandas. Em um trabalho de 1983, Goulding ${ }^{6}$ ainda considera que todo esse processo também é essencial para a prosperidade de uma nação. Back et al. ${ }^{1}$ afirmam que o desenvolvimento de produto compreende os aspectos de planejamento e projeto, ao longo de todas as atividades da sequência do processo, desde a pesquisa de mercado, até o descarte do produto ao final de seu ciclo de vida. Para Rozenfeld et al. ${ }^{2}$, o Processo de Desenvolvimento de Produtos (PDP) situase na interface entre empresa e mercado e deve captar as necessidades deste e propor soluções para suprir as demandas percebidas.

\section{MODELOS REFERENCIAIS DE PDP}

Os modelos de referência para o PDP surgiram para minimizar as limitações da visão desse processo por parte dos atores do desenvolvimento de produtos. Sabe-se que, de maneira geral, as empresas não possuem uma visão unificada desse processo e esse fator pode contribuir com baixas eficiência e eficácia no PDP. Assim, o intuito da adoção de um modelo de referência é formalizar o processo de desenvolvimento de produtos e fazer com que os envolvidos tenham uma visão comum sobre as melhores práticas para que seja dado prosseguimento às etapas do processo ${ }^{2}$. Existem diversos modelos de referência apresentados na literatura e eles podem ser classificados segundo uma série de aspectos diferentes. No entanto, observase que, de maneira geral, existem modelos generalistas e modelos específicos, sendo que estes são aplicados 


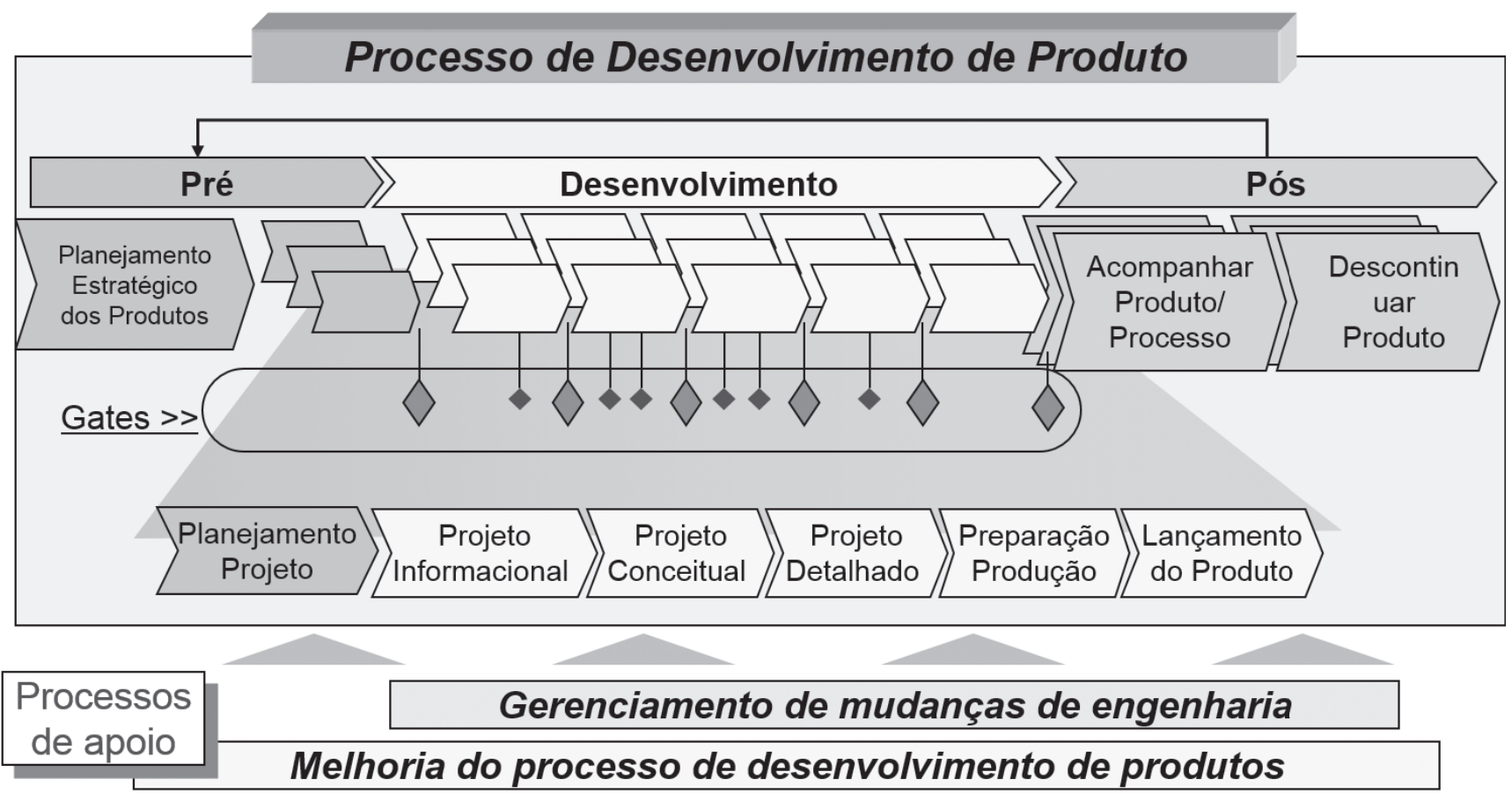

Figura 1. Fases do PDP conforme o Modelo Unificado do PDP $^{2}$.

apenas a alguns setores produtivos e aqueles podem ser flexibilizados conforme cada aplicação ${ }^{2 ; 7}$.

É importante evidenciar que entre os diversos modelos de referência presentes na literatura, o Modelo Unificado de Rozenfeld et al. ${ }^{2}$ (Figura 1) foi desenvolvido pela união das metodologias, estudos de caso, experiências e melhores práticas identificadas por uma equipe com diversos profissionais e pesquisadores atuantes em indústrias do complexo metal-mecânico. Dessa forma, este modelo é considerado como o de referência neste trabalho por possuir aplicação no ramo industrial em estudo.

\section{MODELOS DE MATURIDADE EM PDP}

O termo maturidade transmite a noção de que o desenvolvimento de um produto ou serviço ocorre no sentido de um estágio inicial para um estágio mais avançado, sendo que todo o processo deve percorrer uma série de etapas intermediárias para alcançar a maturidade ${ }^{8}$. O grau de maturidade indica o quanto uma organização aplica as melhores práticas de desenvolvimento de produtos, com foco em desempenhar melhor seu PDP fornecendo pontos de avaliação que podem encaminhar a organização ao longo da melhoria deste $\operatorname{processo}^{2 ;}{ }^{9}$. As primeiras representações do termo maturidade, ainda nos anos de 1950, referiam-se, como exemplo, a necessidades humanas, crescimento econômico e progressão da tecnologia da informação em organizações ${ }^{10}$. Um dos primeiros conceitos de níveis de maturidade foi proposto por Crosby no Aferidor de Maturidade da Gerência de Qualidade, em 1979, sendo que os conceitos deste foram adaptados para outros diferentes modelos de maturidade ao longo dos anos ${ }^{11}$. Entre os diversos modelos de maturidade em PDP presentes na literatura, destacamse o Capability Maturity Model Integration (CMMI), o Organizational Project Management Maturity Model (OPM3) e o Modelo de Maturidade para o PDP, proposto por Rozenfeld ${ }^{2}$.

O Departamento de Defesa dos Estados Unidos (DoD), com vistas a criar condições para a evolução das boas práticas de engenharia de software, patrocinou a fundação do Software Engineering Institute (SEI) em 1984. Posteriormente, a Universidade Carnegie Mellon, responsável pelo SEI, publicou o Capability Maturity 
Model $(\mathrm{CMM}){ }^{12}$. Este modelo tornou-se reconhecido pela comunidade de engenharia de software e, após a integração com outros modelos derivados, encontrase atualmente nomeado Capability Maturity Model Integration (CMMI) versão 1.3. Tal modelo apresenta como áreas de interesse: CMMI para desenvolvimento, CMMI para aquisição e CMMI para serviços ${ }^{12 ;}{ }^{13}$.

Por sua vez, o OPM3, acrônimo de Organizational Project Management Maturity Model é um modelo de maturidade desenvolvido sob a administração do Project Management Institute (PMI) e publicado em sua primeira versão em 2003. O objetivo do modelo é prover uma forma de as organizações entenderem a gestão organizacional de seus projetos e medirem sua maturidade, conforme as melhores práticas recomendadas pelo modelo, e assim desenvolverem seus planos de melhoria na área de gerenciamento de projetos ${ }^{14}$.

O Modelo de Maturidade para o PDP, proposto por Rozenfeld et al. ${ }^{2}$, considera que a empresa deve ser visualizada de forma holística e identifica os elementos estratégia, atividades, informações, recursos e organização, que definem cinco níveis de maturidade:

Nível 1: Básico - Indica que apenas algumas atividades do Modelo Unificado são realizadas. Apresenta quatro subníveis.

Nível 2: Intermediário - As atividades são padronizadas e seus resultados previsíveis. Métodos e ferramentas consagradas de apoio ao PDP são utilizados. Apresentam quatro subníveis.

Nível 3: Mensurável: Engloba a realização de todas as atividades dos níveis anteriores. Somase a utilização de indicadores de desempenho para medir o desempenho de todas as atividades. No entanto, as ações de correção ocorrem de maneira não sistemática.

Nível 4: Controlado: Abrange todos os níveis anteriores. As ações de correção passam a ocorrer de maneira sistemática e integrada aos processos de apoio de gerenciamento de mudanças e melhoria incremental.

Nível 5: Melhoria Contínua: Engloba a realização de todas as atividades dos níveis anteriores. Ocorre a integração, com o próprio PDP, dos seguintes processos: Gerenciamento das mudanças de engenharia, melhoria incremental do PDP e processo de transformação do PDP.
O Modelo de Maturidade para o PDP, proposto por Rozenfeld $e t a l .{ }^{2}$, é utilizado neste trabalho por ter sido desenvolvido para avaliação de maturidade em PDP para o Modelo Unificado de Rozenfeld et al. ${ }^{2}$.

\section{Technology Readiness Level (TRL)}

A escala de maturidade tecnológica Technology Readiness Level (TRL) foi desenvolvida com o intuito de prover uma medida relacionada ao estado de uma nova tecnologia em relação ao seu uso para futuros sistemas espaciais. Consolidou-se como uma métrica de uso mundial importante não apenas para avaliar maturidade tecnológica, mas como método para analisar riscos inerentes ao processo de desenvolvimento tecnológico e fornecer bases para a tomada de decisão e orientações para gestores voltados à Pesquisa e Desenvolvimento ${ }^{15}$; 16 Foi inicialmente concebida em 1974 por Stan Sadin, pesquisador da National Aeronautics and Space Administration (NASA) ${ }^{17}$. Em 1989, a escala foi formalizada, ainda com sete níveis de maturidade ${ }^{18}$ e em 1995 foi reforçada com mais dois níveis, em um espectro de um a nove, conforme ilustra a Figura 2, com definições detalhadas de cada nível e inserção de exemplos de aplicação ${ }^{15 ; 18}$.

O TRL tem como objetivo tornar a avaliação e comunicação do nível de maturidade em novas tecnologias aeroespaciais mais efetiva e facilitar o entendimento dos stakeholders e colaboradores envolvidos em desenvolvimento de projetos da NASA. Ainda, provê um índice de avaliação de maturidade que pode ser comparado entre diferentes tecnologias. No Brasil, podem ser encontradas aplicações do TRL no Instituto Nacional de Pesquisas Espaciais (INPE), no departamento de Desenvolvimento Tecnológico da EMBRAER/S.A. (Empresa Brasileira de Aeronáutica) e na realização das atividades de desenvolvimento tecnológico nas dependências do CCM/ITA (Centro de Competência em Manufatura / Instituto Tecnológico de Aeronáutica) ${ }^{20 ; 21 ; 22 ; 23}$.

Dada a universalidade de aplicações do TRL, foram desenvolvidas escalas derivadas, tais como: Manufacturing Readiness Levels (MRL), Technology Readiness Assessment (TRA), Technology Program Management Model (TPMM), System of Systems Technology Readiness Assessment (SoS TRA), Design Readiness Level (DRL) e Programmatic Readiness Level (PRL) 24; 25; 26; 27. 


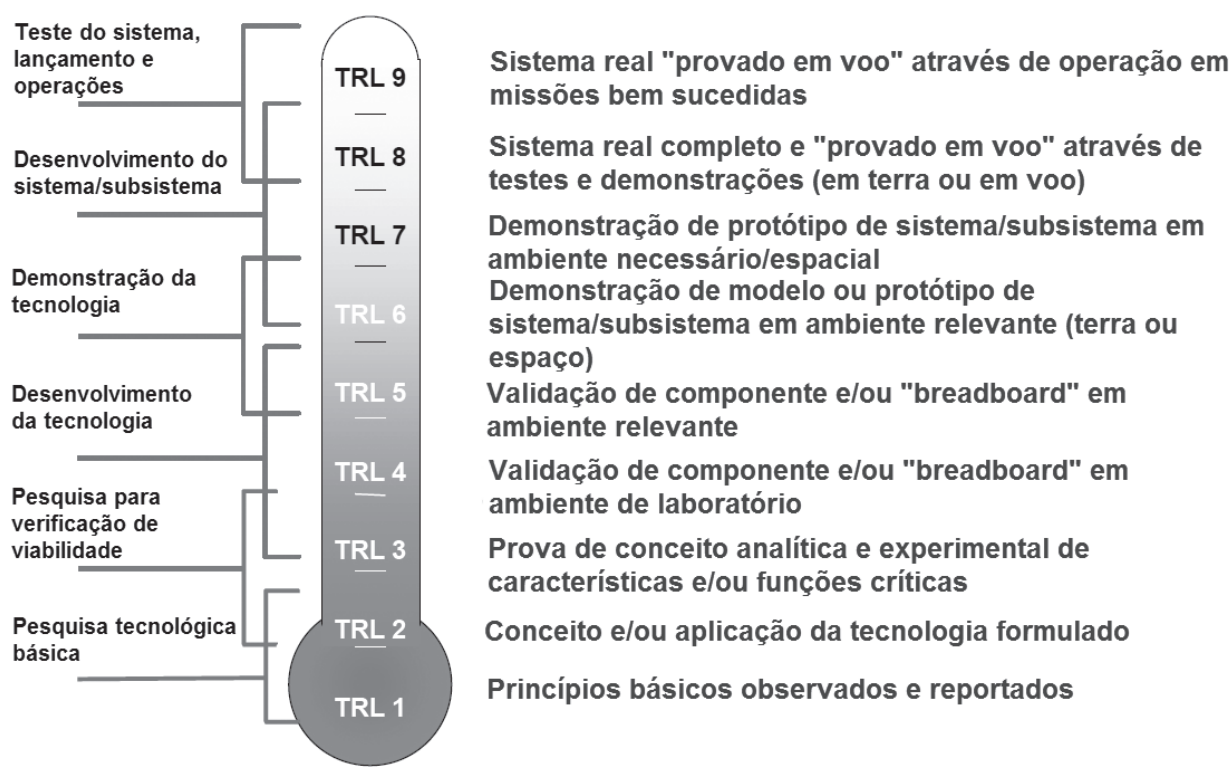

Figura 2. Esquema do "termômetro" da escala de níveis de maturidade do TRL ${ }^{1-9}$.

Este trabalho posiciona-se em relação aos diversos métodos e modelos apresentados com a entrega de um método para medição de maturidade em PDP que integra o modelo de maturidade proveniente do modelo de referência Unificado de Rozenfeld et al. ${ }^{2}$ à escala TRL, utilizada em seu conceito inicial para a medição de maturidade em desenvolvimento de tecnologia aeroespacial. A integração proposta permite que seja realizada a avaliação de maturidade em PDP com o auxílio de uma métrica consagrada pela engenharia aeroespacial. Assim, obtémse o nível de maturidade em PDP, conforme prescreve a escala TRL. Portanto, o benefício desta proposta reside no uso das vantagens do uso do TRL, tais como facilidade de comunicação, fácil entendimento por parte de times de projeto para uma aplicação, maior quantidade de níveis (nove níveis, e não cinco níveis, como existente em outros métodos). Estas vantagens possibilitam uma descrição mais pormenorizada do indicador de maturidade e a própria experiência de usos do TRL em diversas outras áreas e em diversos programas governamentais, militares e industriais. Além disso, a aplicação do método proposto supre a necessidade de conhecimento da maturidade em PDP para as indústrias goianas e permite que essa demanda de informações seja determinada. Ainda, o método proposto será disponibilizado para aplicação também pelos colaboradores dos Institutos SENAI de Tecnologia envolvidos na prestação de serviços tecnológicos, visto que este possui fácil implementação e capacidade de adequação à rotina das atividades de prestação de apoio técnico nos serviços destinados ao desenvolvimento de produtos.

\section{Elaboração do Método}

A modelagem IDEF0 (Integration Definition for Function Modelling) facilita e sistematiza a identificação das funções a serem realizadas e os recursos necessários para que elas sejam cumpridas ${ }^{28} \mathrm{e}$ foi utilizado para formalizar o fluxo de atividades e trazer rigor, exatidão e clareza na elaboração do método para avaliação de maturidade em PDP. A Figura 3 apresenta o primeiro nível hierárquico da modelagem (IDEF0). No lado esquerdo e direito da figura identifica-se, respectivamente, a entrada (input) e as saídas (outputs) para o processo de construção do método para mensuração de maturidade. $\mathrm{Na}$ parte inferior, encontram-se os recursos utilizados para viabilizar a execução do processo. Na parte superior, encontram-se os controles considerados para que a função gere adequadamente as saídas a partir das entradas e dos recursos. 


\section{Artigo Geral 1}

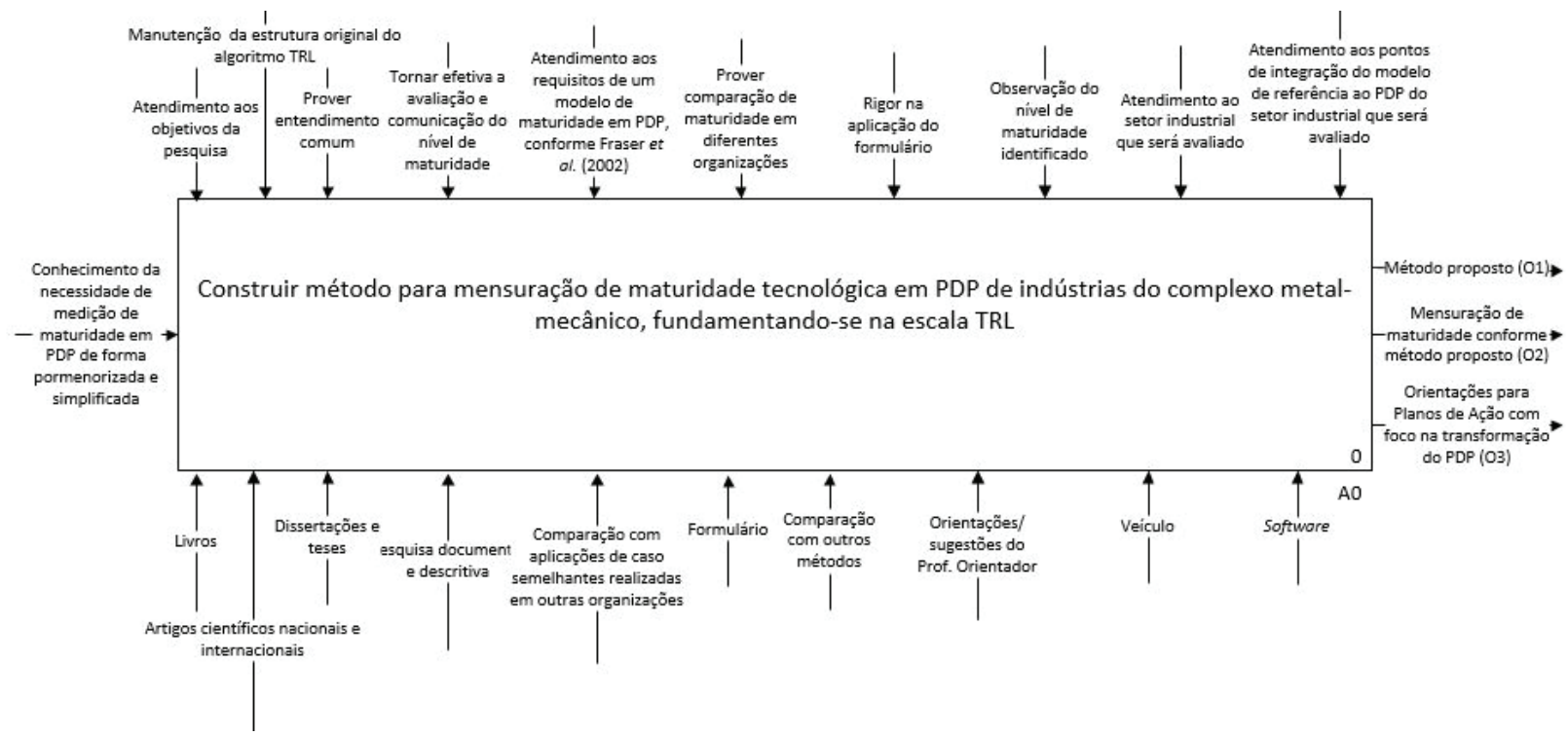

Figura 3. Modelagem IDEF0 utilizada para a construção do método proposto no trabalho.

A Figura 4 mostra o fluxo das atividades principais realizadas na pesquisa, conforme a modelagem IDEF0 aplicada ao segundo nível hierárquico. Essas atividades são descritas em forma de etapas. A primeira etapa descreve o processo de elicitação de métodos, técnicas e ferramentas usadas no trabalho. A segunda ocupa-se com o processo para a proposição do método para mensuração de maturidade em PDP. A terceira etapa apresenta o guia de aplicação do método desenvolvido.
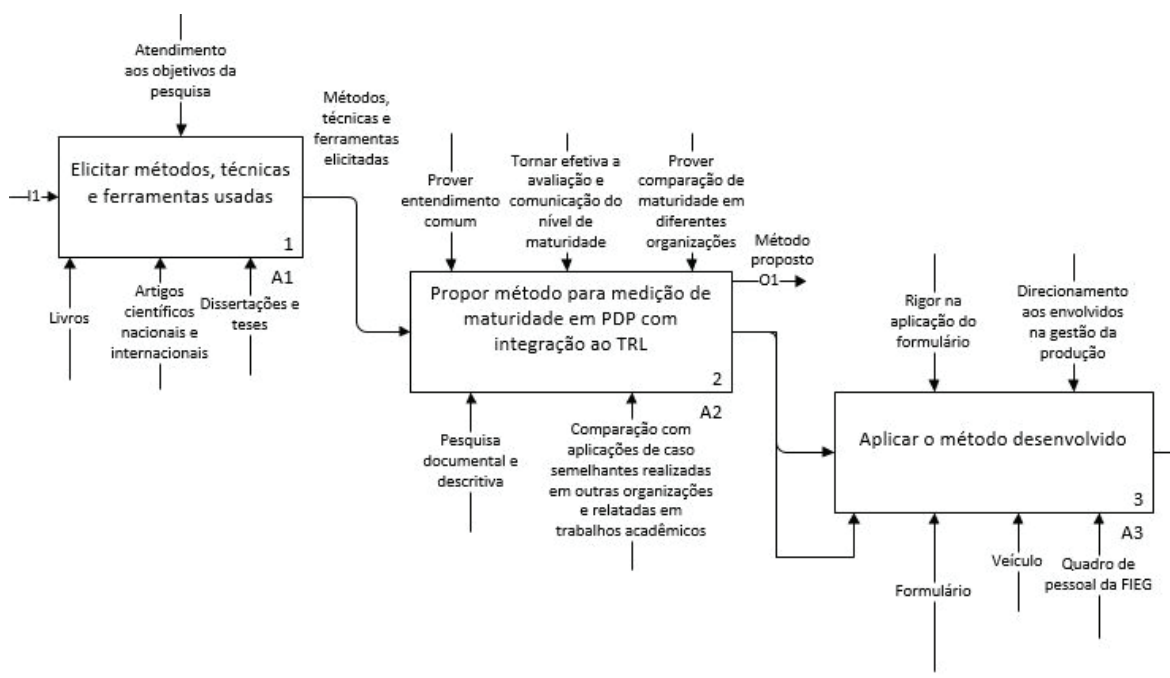

Figura 4. Modelagem IDEF0 no segundo nível de detalhamento.

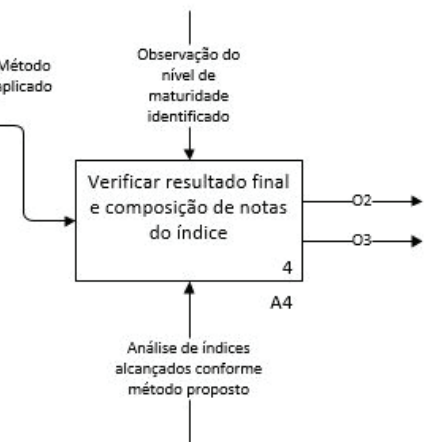


Em relação à etapa <Elicitar métodos, técnicas e ferramentas usadas $>$, buscaram-se informações relacionadas ao conceito, importância e modelos referenciais do PDP, aos métodos para mensuração de maturidade em seus diversos fins e à escala de Nível de Maturidade ou Prontidão Tecnológica (TRL) e seus diversos usos, variantes, algoritmo, formas de aplicação e pontuação de escalas.

Em relação à etapa <Propor método para medição de maturidade em PDP com integração ao TRL $>$, foram consideradas outras etapas secundárias. A Figura 5 apresenta a identificação do modelo de referência em PDP a ser empregado para a integração da escala de medição de maturidade. É utilizado o Modelo Unificado proposto por Rozenfeld et al. ${ }^{2}$ porque esse modelo é genérico (aplicação em diversas empresas diferentes de um mesmo complexo industrial) e usado no desenvolvimento de produtos que envolvem a tecnologia de fabricação mecânica voltada à produção de equipamentos, eletrodomésticos, linha branca, automóveis, entre outros, ou seja, ao PDP do complexo da indústria metal-mecânica que é o foco de aplicação deste trabalho.

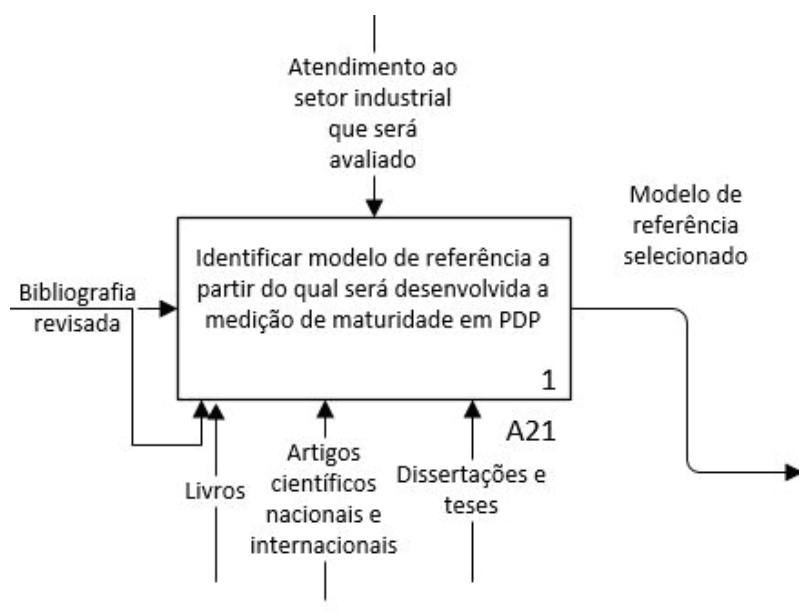

Figura 5. Modelagem IDEF0 do processo A21.

A segunda etapa considerada (Figura 6) abrange a verificação das dimensões de visualização do modelo em relação ao processo de negócio da empresa. O Modelo de referência adotado considera a existência de quatro dimensões: Estratégia, Organização, Atividades e Recursos ${ }^{29}$, sendo que a dimensão importante para a medição de maturidade é a dimensão Atividades.

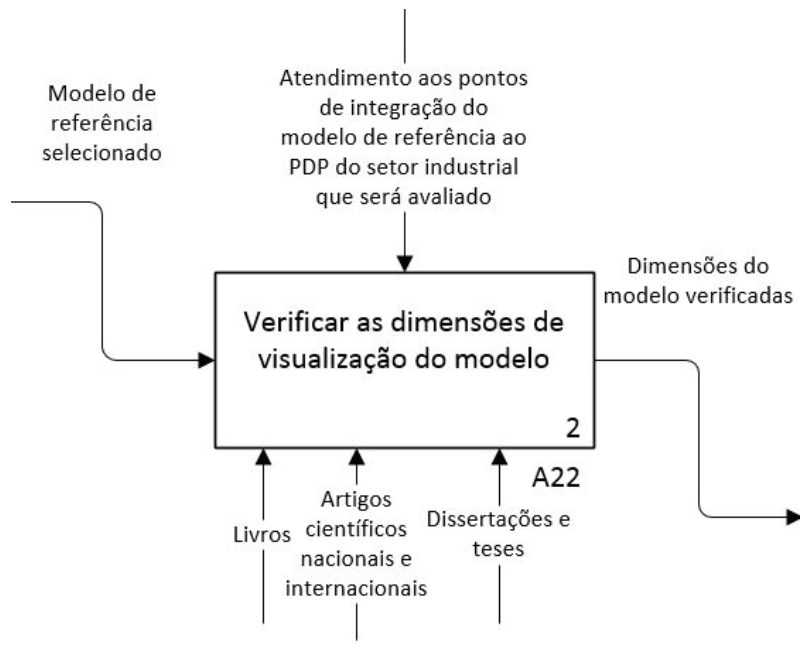

Figura 6. Modelagem IDEF0 do processo A22.

A terceira etapa considerada (Figura 7) compreende a verificação das dimensões para realização da avaliação de maturidade.

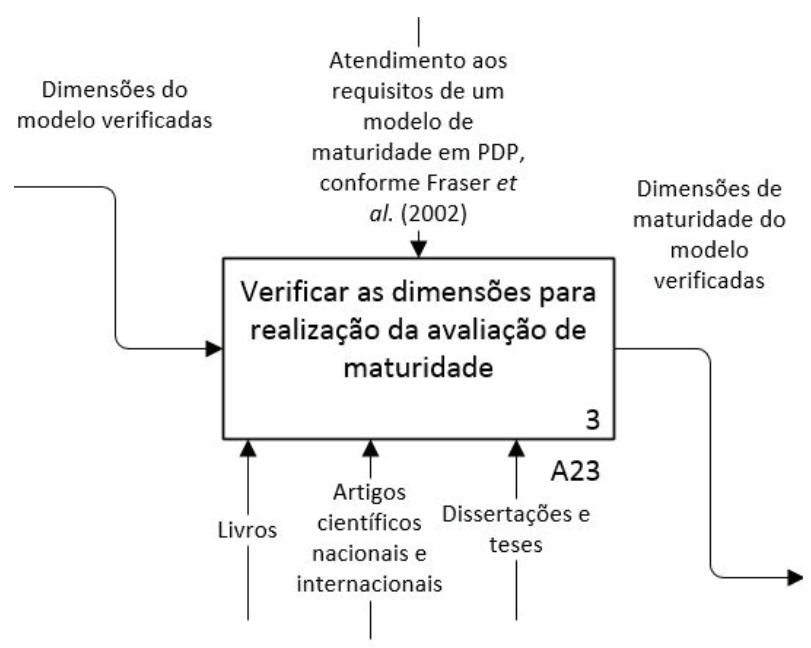

Figura 7. Modelagem IDEF0 do processo A23

A dimensão Atividades do modelo de referência adotado considera a existência de três dimensões para avaliação do grau de maturidade ${ }^{2}$ :

a) Quais atividades propostas no modelo de referência são aplicadas; 
b) Como essas atividades são realizadas (métodos e ferramentas utilizados);

c) Em que etapa do ciclo de evolução (desenvolvimento de produtos com alto, médio ou baixo graus de inovação ou desenvolvimento de produtos Follow source) a empresa se encontra.

Assim, é analisado em qual nível as atividades, métodos e ferramentas estão de fato sendo utilizados. Essas dimensões são integradas à escala TRL e ao método proposto atribui-se a nomenclatura New Product Development Readiness Level - NPDRL (Nível de Prontidão/Maturidade no Processo de Desenvolvimento de Produtos), conforme apresentado no Quadro 1, no qual são observados os requisitos comuns a escalas de maturidades propostos por Fraser et al. ${ }^{8}$.

A integração das escalas TRL e nível de maturidade em PDP ocorre de forma a sistematizar níveis TRL a todo o Processo de Desenvolvimento de Produtos. É importante ressaltar que o TRL refere-se a estágios de desenvolvimento de uma tecnologia aeroespacial em particular, a qual se torna validada plenamente ao atingir o maior patamar da escala, TRL 9 (muito embora apresente potencial real de manufatura a partir do TRL 6). Por sua vez, o nível de maturidade em PDP refere-se ao patamar de sistematização e execução de melhores práticas, conforme um modelo em que uma empresa encontra-se ao executar todas as suas atividades de desenvolvimento de produtos. Assim, quando uma empresa encontra-se no nível 1 de maturidade em desenvolvimento de produtos, infere-se que está desenvolvendo produtos, percorrendo etapas em fluxo de atividades conforme o Modelo Unificado de Rozenfeld et al. ${ }^{2}$; todavia com baixo nível de sistematização e pouca utilização das melhores práticas recomendadas pelo modelo de referência. Fundamentando-se no diagnóstico de maturidade em PDP apresentado, o modelo de referência sugere a realização de um processo de transformação para que a organização alcance um patamar superior na escala de maturidade.

Os níveis da escala NPDRL (Nível de Prontidão no Processo de Desenvolvimento de Produtos) são alinhados à escala TRL conforme nove níveis e estes são limitados conforme os níveis de maturidade em PDP propostos por Rozenfeld et al. ${ }^{2}$. No entanto, os autores consideraram a existência de cinco níveis de maturidade, em que os dois
Quadro 1. Integração TRL com a proposta NPDRL.

\begin{tabular}{|c|c|c|c|}
\hline TRL & Descrição & NPDRL & Descrição \\
\hline 1 & $\begin{array}{l}\text { Princípios básicos } \\
\text { observados e } \\
\text { reportados }\end{array}$ & 1 & $\begin{array}{l}\text { Nível básico de } \\
\text { concepção do } \\
\text { produto (processo } \\
\text { não sistematizado) }\end{array}$ \\
\hline 2 & $\begin{array}{c}\text { Conceito de } \\
\text { tecnologia e/ou } \\
\text { aplicação formulado }\end{array}$ & 2 & $\begin{array}{c}\text { Integração informal } \\
\text { ao planejamento } \\
\text { estratégico } \\
\text { da empresa e } \\
\text { atendimento } \\
\text { informal aos } \\
\text { requisitos dos } \\
\text { clientes }\end{array}$ \\
\hline 3 & $\begin{array}{l}\text { Funções analíticas e } \\
\text { experimentais críticas } \\
\text { e/ou prova de conceito }\end{array}$ & 3 & $\begin{array}{l}\text { Plano de processo } \\
\text { macrorrealizado } \\
\text { sistematicamente } \\
\text { e homologação } \\
\text { do processo de } \\
\text { produção }\end{array}$ \\
\hline 4 & $\begin{array}{c}\text { Validação de } \\
\text { componente e/ou } \\
\text { conjunto em ambiente } \\
\text { de laboratório }\end{array}$ & 4 & $\begin{array}{l}\text { Desenvolvimento } \\
\text { de portfólio de } \\
\text { produtos e propostas } \\
\text { de gestão de } \\
\text { projetos e custos } \\
\end{array}$ \\
\hline 5 & $\begin{array}{c}\text { Validação de } \\
\text { componente e/ou } \\
\text { conjunto em ambiente } \\
\text { relevante }\end{array}$ & 5 & $\begin{array}{c}\text { Realização de } \\
\text { modelagem } \\
\text { funcional do } \\
\text { produtos e uso } \\
\text { de ferramentas } \\
\text { consagradas de } \\
\text { desenvolvimento de } \\
\text { produtos }\end{array}$ \\
\hline 6 & $\begin{array}{l}\text { Modelo de sistema/ } \\
\text { subsistema ou } \\
\text { demonstração de } \\
\text { protótipo em ambiente } \\
\text { relevante }\end{array}$ & 6 & $\begin{array}{l}\text { Gestão de portfólio } \\
\text { integrada ao } \\
\text { gerenciamento } \\
\text { estratégico da } \\
\text { empresa e desenho } \\
\text { dos processos } \\
\text { de negócio } \\
\text { relacionados ao PDP }\end{array}$ \\
\hline 7 & $\begin{array}{l}\text { Demonstração de } \\
\text { protótipo no ambiente } \\
\text { operacional esperado }\end{array}$ & 7 & $\begin{array}{l}\text { Integração com } \\
\text { parceiros da cadeia } \\
\text { de suprimentos } \\
\text { uso de sistemas de } \\
\text { gerenciamento de } \\
\text { dados de produtos }\end{array}$ \\
\hline 8 & $\begin{array}{l}\text { Sistema real completo } \\
\text { e qualificado por } \\
\text { meio de testes e } \\
\text { demonstração }\end{array}$ & 8 & $\begin{array}{c}\text { Integração } \\
\text { completa de } \\
\text { gestão de projetos } \\
\text { e consideração } \\
\text { do conceito de } \\
\text { desenvolvimento } \\
\text { sustentável }\end{array}$ \\
\hline 9 & $\begin{array}{l}\text { Prova de operação do } \\
\text { sistema real por meio } \\
\text { de execuções bem } \\
\text { sucedidas }\end{array}$ & 9 & $\begin{array}{l}\text { Melhoria contínua } \\
\text { com a integração } \\
\text { de indicadores de } \\
\text { desempenho em } \\
\text { todas as atividades } \\
\text { para efetuar } \\
\text { correções no PDP }\end{array}$ \\
\hline
\end{tabular}


primeiros desdobram-se em outros níveis identificados. $\mathrm{Na}$ escala NPDRL não se considera a existência de subníveis e todas as indicações de níveis dos autores citados se alinham segundo uma escala com nove níveis. Atenção especial deve ser dada ao fato de que os níveis três, quatro e cinco do modelo de Rozenfeld et al. ${ }^{2}$ correspondem aos "níveis avançados de maturidade" 2, p.488 em que se parte da premissa de que a empresa padronizou as atividades de desenvolvimento de produtos e utiliza métodos e ferramentas consagrados no PDP. Dessa forma, na escala NPDRL, esses níveis avançados correspondem ao nível NPDRL ${ }^{9}$, adequando-se assim os níveis de maturidade do modelo de referência ao método apresentado neste trabalho. Deve-se ressaltar ainda que os níveis de maturidade do modelo de referência têm sua divisão realizada conforme o conhecimento e experiência de Rozenfeld et al. ${ }^{2}$ e são consistentes com a proposta apresentada pelos autores. Assim, os limites de cada nível e subnível de maturidade em PDP são mantidos no método NPDRL e o mapeamento das atividades, métodos, ferramentas e ciclo incremental de evolução da organização ligam-se aos níveis NPDRL com a aplicação de um algoritmo semelhante ao desenvolvido pela Air Force Research Laboratory (AFRL) para determinação do índice de avaliação TRL. Essa atividade compõe a quarta etapa para desenvolvimento do NPDRL.

A quarta etapa (Figura 8) considerada abrange a adaptação das dimensões de maturidade ao algoritmo do TRL, com atenção à manutenção da estrutura desse algoritmo. São utilizados os recursos de planilha eletrônica em Microsoft Excel ${ }^{\circledR}$, artigo relacionado ao algoritmo do TRL de Nolte et al. ${ }^{30}$, relatórios de uso do TRL nos Departamentos de Energia e Defesa dos Estados Unidos da América e discussões com especialistas da área de PDP.

É importante considerar que, nessa fase, a saída é o método de medição de maturidade proposto, com um "termômetro" integrado à planilha de análise de maturidade e com o índice de escala para a maturidade da empresa, conforme o modelo de maturidade de Rozenfeld et al. ${ }^{2}$.

Como forma de agilizar o processo de avaliação de maturidade, assim como ocorreu com o TRL com o desenvolvimento do Technology Readiness Calculator, uma planilha eletrônica desenvolvida pelo $\mathrm{AFRL}^{30}$, foi

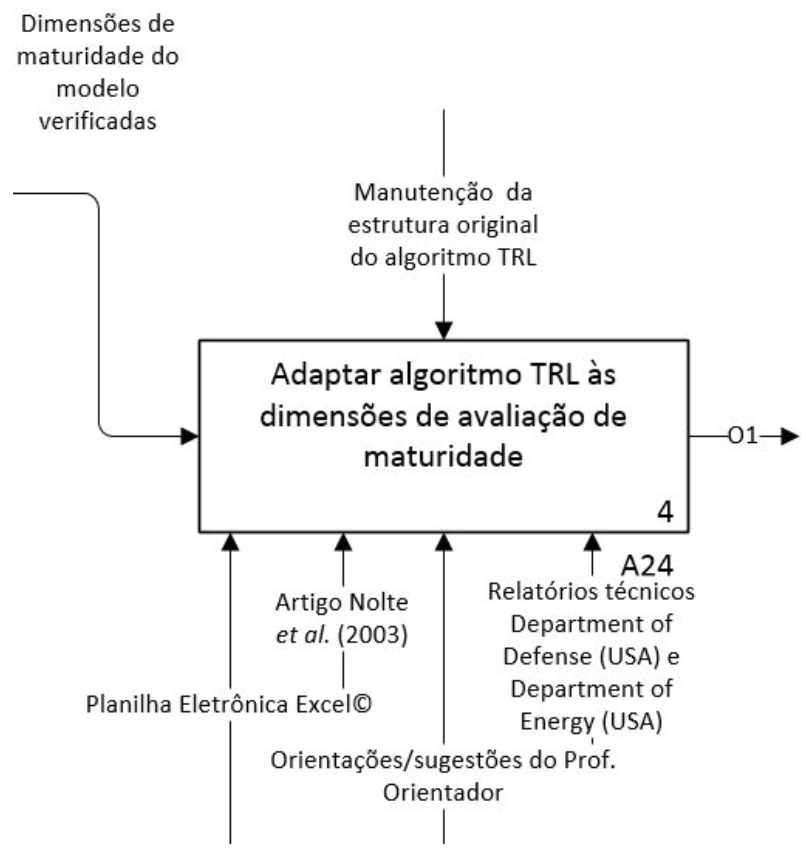

Figura 8. Modelagem IDEF0 do processo A24.

desenvolvida uma planilha eletrônica em que é possível lançar dados de questionários preenchidos com visita à empresa e a geração de indicação em um "termômetro NPDRL" com lógica semelhante ao Technology Readiness Calculator. Ainda, podem ser obtidos dados para avaliações parciais relacionadas à sistematização de atividades, ferramentas e métodos usados em PDP.

O algoritmo NPDRL está implementado em planilha eletrônica denominada NPDRL Calculator 1.0 (Figura 9).

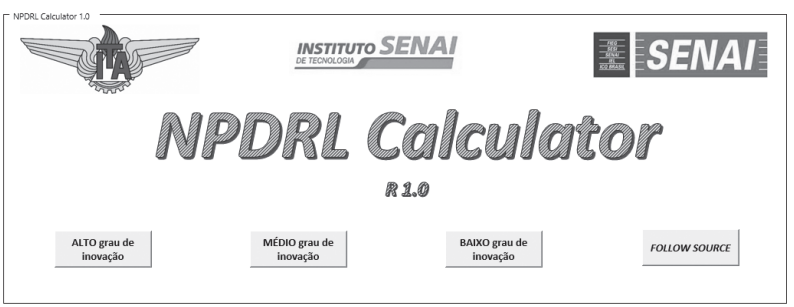

Figura 9. Tela inicial do NPDRL Calculator 1.0. 
A avaliação do algoritmo visa estabelecer o nível de maturidade em PDP com uso do método NPDRL. Esse objetivo é alcançado com a execução do algoritmo em uma estrutura condicional e o resultado é apresentado em um "termômetro" com nove níveis (Figura 10), que podem ser preenchidos com as cores:

a) Verde: o nível proposto foi alcançado;

b) Amarelo: o nível não alcançou pontuação para aprovação pois ainda há atividades não realizadas e métodos/ferramentas consagrados não utilizados de forma frequente pela organização; no entanto, encontra-se próximo à pontuação de aprovação e determinação do nível indicado;

c) Vermelho: alguma pontuação relacionada a atividades e métodos/ferramentas consagrados foi alcançada neste nível ou superiores, no entanto, não possuem frequência de utilização suficiente para o estabelecimento deste nível de maturidade.

d) Branco: nenhuma pontuação foi alcançada nesse nível ou superior.

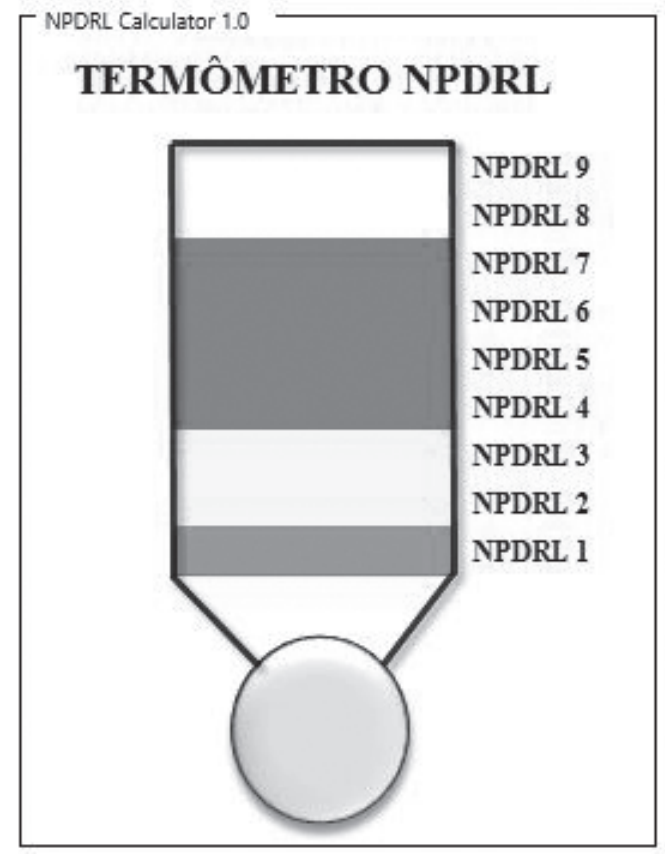

Figura 10. Exemplo de Termômetro NPDRL obtido NPDRL Calculator 1.0.
Em uma situação de uma avaliação fictícia, conforme representado na Figura 10, a organização atingiu suficiência apenas no nível de maturidade 1 . Nos níveis 2 e 3, não alcançou suficiência, mas obteve pontuação próxima à aprovação. Nos níveis 4, 5, 6 e 7 executa alguma das atividades ou métodos propostos no modelo de referência e nos níveis 8 e 9 atingiu nenhuma pontuação. Neste exemplo, pode-se inferir que a empresa tem seu PDP organizado com um baixo nível de sistematização. Rozenfeld et al. ${ }^{2}$, por sua vez, afirmam que em uma aplicação de caso de avaliação de maturidade é possível uma empresa executar práticas pertencentes a níveis de maturidade diferentes ao mesmo tempo. Apesar disso, a divisão em níveis de maturidade proposta pelos autores (e usada neste trabalho) é consistente pois considera uma proposta lógica de passos evolutivos do PDP.

Deve ser observado que para a obtenção de dados para alimentação do NPDRL Calculator 1.0 é realizada uma avaliação com colaboradores da organização relacionados diretamente à gestão do PDP. Suas percepções e constatações são colhidas pela planilha com o apoio de um questionário em que as respostas encontram-se em forma de Escala Likert ${ }^{31}$ de cinco pontos, que permite realização de avaliação psicométrica assim como ocorre no processo de determinação do TRL.

As outras fases a serem consideradas para efetivação do método proposto neste trabalho são a $<$ Aplicação do método desenvolvido $>$ e a $<$ Verificação do resultado final e composição de notas do índice de maturidade $>$ como mostrado na Figura 4. No entanto, essas etapas visualizam o processo de aplicação do método NPDRL em empresas do ramo metal-mecânico e não se constituem como escopo deste trabalho. O ponto principal a ser observado nessas fases é que a aquisição de dados para avaliação de maturidade ocorre com visita in loco à empresa em que se pretende avaliar o índice NPDRL. Para isso, deve ser usado um questionário relacionado aos níveis NPDRL que deve preenchido presencialmente por um colaborador diretamente envolvido com a gestão do PDP da organização.

\section{Considerações Finais}

O objetivo deste trabalho foi propor um método para mensuração do grau de maturidade no PDP, fundamentado na escala TRL (Technology Readiness 
Level), de forma a possibilitar a geração de conhecimento sobre o grau de maturidade do PDP em indústrias do complexo metal-mecânico e assim indicar um norteador para o encaminhamento de orientações para planos de ação a serem propostos durante a execução de possíveis serviços dos ISTs goianos. Este objetivo foi alcançado com a determinação do método NPDRL proposto.

$\mathrm{O}$ resultado mais significativo desta pesquisa referese ao desenvolvimento da escala de maturidade NPDRL. Embora esta escala fundamente-se em outro método para avaliação de maturidade do PDP, a contribuição encerra-se na junção desses conceitos de avaliação com os níveis de níveis de maturidade de escala TRL, que é normalmente usada na análise de tecnologias da engenharia aeroespacial. Dessa forma, os benefícios do uso do TRL, entre os quais se reitera a universalidade de conhecimento de níveis, capacidade de comunicação e facilidade de compreensão por parte dos times foram adicionados à mensuração de maturidade em PDP. Ainda, o NPDRL apresenta maior quantidade de níveis em relação aos modelos de maturidade em PDP propostos na literatura. Além disso, como forma de propiciar futuras aplicações do método pelos colaboradores dos IST goianos, desenvolveu-se uma planilha eletrônica (NPDRL Calculator 1.0) em que está implementado o algoritmo e um "termômetro" responsável por indicar o nível de maturidade NPDRL.

A aplicação deste método possibilitará aos colaboradores dos ISTs o conhecimento do nível de maturidade em PDP de uma organização ao realizarem serviços técnicos voltados ao desenvolvimento de produtos em indústrias goianas e em outra situação, é possível selecionar empresas para a oferta de determinados serviços e consultorias específicas nesta linha de ação. Assim, estas propostas de desenvolvimentos futuros poderão ser realizadas com a aplicação do método NPDRL em empresas do parque industrial goiano.

\section{Referências}

1. BACK, N.; OGLIARI, A.; DIAS, A.; SILVA, C. D. Projeto Integrado de Produtos: Planejamento, concepção e modelagem. Barueri: Manole, 2008. 648 p.

2. ROZENFELD, H.; FORCILLINI, F. A.; AMARAL, D. C.; TOLEDO, J. C. D.; SILVA, S. L. D.; ALLIPRANDINI, D. H.; SCALICE, R. K. Gestão de Desenvolvimento de Produto: Uma referência para a melhoria do processo. São Paulo: Saraiva, 2006. 576 p. p.

3. ULRICH, K. T.; EPPINGER, S. D. Product design and development. New York: McGraw-Hill Education, 2003. p.

4. KAMINSKI, P. C.; DE OLIVEIRA, A. C.; LOPES, T. M. Knowledge transfer in product development processes: A case study in small and medium enterprises (SMEs) of the metalmechanic sector from São Paulo, Brazil. Technovation, v. 28, n. $1-2$, p. 7, 2008

5. IMB, I. M. B. D. E. E. E. S. Pesquisa de Intenção de Investimentos no Estado de Goiás. Secretaria de Estado de Gestão e Planejamento. Goiânia, 8 p. 2014.

6. GOULDING, I. New Product Development: A Literature Review. European Journal of Marketing, v. 17, p. 27, 1983.

7. JUNIOR, C. A. C. Proposta de um métodos de análise de maturidade e priorização de melhorias na gestão do PDP. 2008. 184 p. (Mestrado). Engenharia de Produção, Universidade Federal do Rio Grande do Sul, Porto Alegre.

8. FRASER, P.; MOULTRIE, J.; GREGORY, M. The use of maturity models/grids as a tool in assessing product development capability. Engineering Management Conference, p. 5, 2002.

9. DOOLEY, K.; SUBRA, A.; ANDERSON, J. Maturity and its impact on new product development project performance. Research in Engineering Design, v. 13, n. 1, p. 6, 2001.

10. RÖGLINGER, M.; PÖPPELBUß, J.; BECKER, J. Maturity models in business process management. Business Process Management Journal, v. 18, n. 2, p. 18, 2012.

11. QUINTELLA, H. L. M. D. M.; ROCHA, H. M. Nível de maturidade e comparação dos PDPs de produtos automotivos. Production, v. 17, p. 16, 2007.

12. MIYASHIRO, M. A. S. Uma ferramenta de apoio ao diagnóstico do nível de maturidade utilizando um modelo de rede neural. 2007. 104 (Mestrado). Instituto de Pesquisas Espaciais INPE, São José dos Campos.

13. SEI, S. E. I. CMMI for Development, Version 1.3 (CMU/ SEI-2010-TR-033). Carnegie Mellon University. Pittsburgh, Pennsylvania, 482 p. 2010.

14. PMI, P. M. I. Organizational Project Management Maturity Model (OPM3) Knowledge Foundation. Newtown Square, Pennsylvania, USA: PMI, 2003. 195 p.

15. MANKINS, J. C. Technology readiness assessments: A retrospective. Acta Astronautica, v. 65, p. 7, 2009

16. ALTUNOK, T.; CAKMAK, T. A technology readiness levels (TRLs) calculator software for systems engineering and technology management tool. Advances in Engineering Software, v. 41, n. 5 , p. $9, \mathbf{2 0 1 0}$

17. NASA, N. A. A. S. A. Technology Readiness Levels Demystified 2010. Disponível em: < http://www.nasa.gov/topics/aeronautics/ features/trl_demystified.html >. Acesso em: 17 abr. 2015. 
18. SADIN, S. R.; POVINELLI, F. P.; ROSEN, R. The NASA technology push towards future space mission systems. Acta Astronautica, v. 20, n. 0, p. 4, 1989.

19. NASA, N. A. A. S. A. HRST Technology Assessments. 2000. Disponível em: $<$ http://www.hq.nasa.gov/office/codeq/trl/trlchrt. pdf $>$. Acesso em: 20 mar. 2015.

20. INPE, I. N. D. P. E. Roteiro de Desenvolvimento de Missões e Tecnologias Espaciais para o período 2008-2020 - Roteiro MTE 230 p. 2008.

21. ITA, I. T. D. A. Tecnologia de alinhamento e nivelamento automatizados. 2015. Disponível em: < http://www.ita.br/ noticias/lam >. Acesso em: 19 mai. 2015.

22. CNI, C. N. D. I. Inovação em cadeias de valor de grandes empresas: 22 casos. CNI: IEL. Brasília, 464 p. 2013.

23. ARAUJO, C. S. Lessons learned on the planning and execution of technology innovation projects with academic partnership: aerospace industry case study. Product: Management \& Development, v. 10, p. 10, 2012.

24. PHILLIPS, E. L. The development and initial evaluation of the Human Readiness Level framework. 2010. 171 Dissertação de Mestrado (Mestrado). Naval Postgraduate school, Monterey, California

25. ALMEIDA, V. R. D. Sensores inerciais fotônicos para aplicações aeroespaciais: nível de maturidade tecnológica. 2008. 177 Divisão de Ensino, Escola de Comando e Estado-Maior da Aeronáutica, Rio de Janeiro.

26. HOBSON, B. A Technology Maturity Measurement System for the Department of National Defence Defence: The TML System. Defence Research and Development Canada. Canada, 44 p. 2006.

27. DOD, D. O. D. Manufacturing Readiness Level (MRL) Deskbook. 82 p. 2012

28. KIM, S.-H.; JANG, K.-J. Designing performance analysis and IDEF0 for enterprise modelling in BPR. International Journal of Production Economics, v. 76, n. 2, p. 12, 2002.

29. ROZENFELD, H.; RENTES, A. F.; PORTO, A. J. V.; BREMER, C. F.; AMARAL, D. C.; ALLIPRANDINI, D. H.; SANTOS, E. M. D.; PIRES, E. C.; PALMA, J. G.; OLIVEIRA, J. F. G. D.; CARVALHO, J. D.; TOLEDO, J. C. D.; ORTEGA, L. M.; CARPINETTI, L. C. R.; MAGNANI, M.; MUSETTI, M. A.; SOUZA, M. P. D.; COELHO, R. T.; SILVA, S. L. D.; PIRES, S. R. I.; REZENDE, S. O.; ROMA, W. N. L. Fábrica do futuro: entenda hoje como sua indústria vai ser amanhã. 1 ed. São Paulo: Editora Banas, 2000. 138 p. p.

30. NOLTE, W. L.; KENNEDY, B. C.; DZIEGIEL, R. J. Technology Readiness Calculator. 6th Annual System Engineering Conference 2003.

31. VIEIRA, K. M.; DALMORO, M. Dilemas na Construção de Escalas Tipo Likert: o Número de Itens e a Disposição Influenciam nos Resultados? XXXII Encontro da ANPAD. Rio de Janeiro: 16 p. 2008.

\section{Almiro Martins da Silva \\ Neto ${ }^{1 *}$ \& Luís Gonzaga Trabasso $^{2}$}

\author{
${ }^{1}$ Faculdade de Tecnologia SENAI Roberto Mange, Anápolis, Goiás, \\ Brasil. \\ ${ }^{2}$ Instituto Tecnológico de Aeronáutica, São José dos Campos, São \\ Paulo, Brasil. \\ *E-mail: almiro.senai@sistemafieg.org.br
}

